\title{
Identification of alternative transcripts of rat CD9 expressed by tumorigenic neural cell lines and in normal tissues
}

\author{
Sonja Wolfahrt ${ }^{1}$, Sandra Herman ${ }^{1}$, Claus-Jürgen Scholz ${ }^{2}$, Georg Sauer ${ }^{3}$ and Helmut Deissler ${ }^{1}$ \\ ${ }^{1}$ Department of Obstetrics and Gynaecology, University of Ulm, Ulm, Germany. \\ ${ }^{2}$ IZKF Laboratory for Microarray Applications, University of Würzburg, Würzburg, Germany. \\ ${ }^{3}$ Department of Obstetrics and Gynaecology, Robert Bosch Hospital, Stuttgart, Germany.
}

\begin{abstract}
CD9 is the best-studied member of the tetraspanin family of transmembrane proteins. It is involved in various fundamental cellular processes and its altered expression is a characteristic of malignant cells of different origins. Despite numerous investigations confirming its fundamental role, the heterogeneity of CD9 or other tetraspanin proteins was considered only to be caused by posttranslational modification, rather than alternative splicing. Here we describe the first identification of CD9 transcript variants expressed by cell lines derived from fetal rat brain cells. Variant mRNA-B lacks a potential translation initiation codon in the alternative exon 1 and seems to be characteristic of the tumorigenic BT cell lines. In contrast, variant mRNA-C can be translated from a functional initiation codon located in its extended exon 2, and substantial amounts of this form detected in various tissues suggest a contribution to CD9 functions. From the alternative sequence of variant $\mathrm{C}$, a different membrane topology ( 5 transmembrane domains) and a deviating spectrum of functions can be expected.
\end{abstract}

Keywords: CD9, tetraspanin, transcript, splice variant, membrane topology.

Received: November 15, 2012; Accepted: April 9, 2013.

\section{Introduction}

CD9 is the prototype member of the tetraspanin protein family consisting of two extracellular loops and short intracellular ends that are linked through four transmembrane domains. Tetraspanins play a special role in biological membranes as adaptor proteins in functional complexes with adhesion molecules and other components that are implicated in various cell types in the regulation of fundamental processes, like cell differentiation, proliferation, motility and invasion (Maecker et al., 1997; Hemler, 2005). For many types of malignant diseases, e.g. breast, colon, lung, and cervical cancers (Higashiyama et al., 1995; Miyake et al., 1996; Mori et al., 1998; Sauer et al., 2003), the level of expression of CD9 was found to correlate, in most cases inversely, with the stage of tumor progression, appearance of metastases, or poor clinical prognosis (Romanska and Berditchevski, 2011; Wang et al., 2011). In the fetal and adult rat brain, CD9 was predominantly detected in distinct regions. It is a component of plasma membranes of all types of mature and premature glial cells with a pronounced expression by oligodendrocytes and, in the peripheral nervous system (PNS), Schwann cells (Tole and Patterson, 1993; Anton et al., 1995; Kaprielian et al., 1995; Deissler et al.,

Send correspondence to Helmut Deissler. Department of Obstetrics and Gynaecology, University of Ulm, Frauensteige 14, D-89075 Ulm, Germany. E-mail: helmut_deissler@web.de.
1996; Terada et al., 2002). In the central nervous system and the PNS, CD9 was found concentrated in axoglial paranodal junctions (Ishibashi et al., 2004). Nakamura et al. (1996) confirmed that CD9 is a constituent of myelin and suggested an important role in the regulation of myelination. An initial investigation of human brain tumors did not show a typical inverse, but a positive correlation of CD9 expression with the malignancy of astrocytic tumors, whereas CD9 seemed down-regulated in other types of gliomas compared to their corresponding normal cellular counterparts (Kawashima et al., 2002). In contrast, most malignant neural rat cell lines established from fetal brain cells after in vivo-exposure to ethylnitrosourea strongly expressed CD9. Only two of these cell lines (BT8Ca and BT12Ca) were CD9-negative or showed minimal CD9specific immunoreactivity assessed by flow cytometry (Deissler et al., 1996). Further analyses of these cells, however, indicated predominant expression of different CD9 species that were not detectable with the previously used antibodies. Subsequently, two novel CD9 transcripts containing alternative first exons were identified. Whereas CD9 mRNA-B seems to be a characteristic of BT cell lines, mRNA-C was found to be expressed in various normal tissues. The deduced amino acid sequence, beginning with a functional translation initiation codon, suggested altered membrane topology and functions of this CD9 variant. 


\section{Materials and Methods}

\section{Cell lines and primary antibodies}

Rat neuroectodermal cell lines BT4Ca, BT8Ca and BT12Ca (Laerum et al., 1977) from the collection at the Institute of Cell Biology, University of Essen (Germany) were cultivated in DMEM $/ 10 \%$ FCS (PAA Laboratories, Linz, Austria). Testing of all cultivated cells lines for a potential mycoplasma infection was performed regularly with a Mycoplasma Detection Kit (Roche Diagnostics, Mannheim, Germany).

To detect CD9, we used the monoclonal antibodies MEM-61 (Exbio, Praha, Czech Republic) and NCA-1 (Deissler et al., 1996). In all experiments, specificity of these antibodies was confirmed by parallel processing with an isotype-matched control antibody from Southern Biotechnology (Birmingham, U.S.A).

\section{Immunofluorescence staining}

Cells were seeded onto glass coverslips and cultivated in DMEM $/ 10 \%$ FCS for 2 days. After washing twice with PBS, cells were kept in cold buffered paraformaldehyde ( $4 \%$ solution, pre-cooled in ice) for $10 \mathrm{~min}$. Cells were washed $3 \mathrm{x}$ with PBS, as between all of the following steps: Blocking by $3 \%$ BSA/PBS for 30 min at room temperature (RT), incubation with the CD9-specific antibody (hybridoma supernatant or purified antibody diluted to $0.5 \mu \mathrm{g} / \mathrm{mL}$ in blocking solution) at $4{ }^{\circ} \mathrm{C}$ for $16 \mathrm{~h}$, incubation with the detection antibody goat anti-mouse-AlexaFluor594 (Invitrogen; $2.5 \mu \mathrm{g} / \mathrm{mL}$ in 3\% BSA/PBS) for $1 \mathrm{~h}$ at RT, and mounting with Vectashield Fluorescence Mounting Medium (Vector, Burlingame, UK). Specimens were examined under an Olympus BX51 fluorescence microscope.

\section{Solubilization and Western blot analyses of EGFP fusion proteins}

Cells were collected in phosphate-buffered saline (PBS) and solubilized at a concentration of $6 \times 10^{6}$ cells $/ \mathrm{mL}$ for $1 \mathrm{~h}$ at $4^{\circ} \mathrm{C}$ in $40 \mathrm{mM}$ TrisCl, $\mathrm{pH} 7.4,150 \mathrm{mM}$ $\mathrm{NaCl}$ (TBS), $50 \mathrm{mM}$ n-Octylglucosid (Calbiochem), supplemented with protease inhibitors (Complete mix, Roche Diagnostics, Mannheim). After centrifugation at $15,000 \mathrm{~g}$, the supernatant was analysed by standard polyacrylamide gelelectrophoresis (Laemmli 1970). Proteins were transferred to polyvinylidene difluoride membranes (Pall, Dreieich, Germany) by semi-dry electroblotting for $60 \mathrm{~min}$ at $1.5 \mathrm{~mA} / \mathrm{cm}^{2}$. Membranes were blocked in blocking solution (Pierce, Bonn, Germany) and incubated with a purified polyclonal rabbit anti-EGFP antibody (Abcam, Cambridge, UK; diluted 1:2000 in blocking solution). Bound primary antibodies were detected with a SuperSignal Detection Kit (Pierce) containing a peroxidase-conjugated anti-rabbit IgG antibody and a chemoluminescence substrate.

\section{RT-PCR and RACE}

RNA was isolated with an RNeasy Mini Kit (Qiagen, Hilden, Germany) and residual genomic DNA was removed with a DNA-free Kit (Ambion, Woodward, USA). cDNA to be used in conventional PCRs was synthesized with Multiscribe reverse transcriptase and an oligo-dT primer (Applied Biosystems, Foster City, USA) according to the supplier's protocol. cDNAs representing transcripts from various rat organs were purchased from Clontech (Heidelberg, Germany). For conventional PCR with $50 \mathrm{ng}$ template cDNA, 10 pmol of each primer (Thermo, Ulm, Germany) and Fast Start Taq polymerase (Roche Diagnostics, Mannheim, Germany), the cycling conditions were: $94{ }^{\circ} \mathrm{C}$ for $3 \mathrm{~min}$ followed by 30 cycles of $94^{\circ} \mathrm{C}$ for $30 \mathrm{~s}$, $56{ }^{\circ} \mathrm{C}$ for $30 \mathrm{~s}$ and $72{ }^{\circ} \mathrm{C}$ for $1 \mathrm{~min}$.

Rat CD9-specific primers were: CD9-A-F1 (5'-TGT ACCATGCCGGTCAAAGGAG-3'), CD9-A-F2 (5'-TCC TCTTGGTGATATTCGCCATTG-3'), CD9-A-FNC (5'TGGCACTTTTTAAAAGTGGAGCCTC-3'), CD9-A-R1 (5'-ACTCTAGACCATTTCTCGGCTCCTG-3'), CD9A-R2 (5'-CTCCTGGAGTCTTTAATCACCTCGTC-3'), CD9-A-RNC (5'-GTATCAAATTGTCTTCAATATAAC TTACAACC-3'). Primers specific for the newly identified CD9 transcripts were: rCD9-B-F1 (5'-GCCCAGATCTG TGTCCTGCAC-3') and rCD9-C-F1 (5'-CTGAACGAAA TGATGCCGAAGTCC-3'). In RT-PCR analyses, these were combined with the CD9-A reverse primers.

For amplification of the 5'-ends of CD9 mRNA species, we used the SMART RACE Kit from Clontech (Heidelberg, Germany). cDNA synthesis was performed with a modified oligo(dT) primer and a SMART oligonucleotide resulting in a 5'-extension after (dC)-tailing. The 5'-ends were then amplified by PCR ( 30 cycles of $94^{\circ} \mathrm{C}$ for $30 \mathrm{~s}$, $68^{\circ} \mathrm{C}$ for $30 \mathrm{~s}$ and $72{ }^{\circ} \mathrm{C}$ for $30 \mathrm{~s}$ ) with universal primers binding to the $5^{\prime}$-extension and a $\mathrm{CD} 9$-specific reverse primer (CD9-GSP1: 5'-TTTCCCGCTGGGGCTCATCC TTGTTCC-3'). PCR products were used as a template in a second PCR $\left(25\right.$ cycles of $94^{\circ} \mathrm{C}$ for $30 \mathrm{~s}, 63{ }^{\circ} \mathrm{C}$ for $30 \mathrm{~s}$ and $72{ }^{\circ} \mathrm{C}$ for $30 \mathrm{~s}$ ) with a provided nested universal primer and a nested CD9-specific primer (5'-GCGGCGATCTCAA TGGCGAATATCAC-3'). After analysis by agarose gel electrophoresis, bands were excised and the PCR products isolated with the QIAquick Gel Extraction Kit (Qiagen, Hilden, Germany) for sequence analyses (Seqlab, Göttingen, Germany).

\section{Generation and transfection of expression plasmids}

The cDNAs encoding CD9 species with alternative exons (rCD9-B, rCD9-C with or without terminating stop codons) were amplified by conventional PCR with primers containing extensions that allowed their recombinase-mediated insertion into the donor vector $\mathrm{pDNR}$-Dual with the InFusion recombinase (Clontech, Heidelberg, Germany) according to the suppliers' instructions. Inserts were then transferred to the acceptor vector pLP-EGFP-C1 in a reac- 
tion with Cre recombinase (Clontech) under recommended conditions. Lipofectamine 2000 (Invitrogen, Leck, Netherlands) was used to transfect the purified (EndoFree Plasmid Maxi Kit, Qiagen, Hilden, Germany) plasmids into BT4Ca cells. Fusion proteins with C-terminal EGFP were visualised directly by fluorescence microscopy or by Western blot analyses.

\section{Sequence-deduced prediction of membrane topology}

The membrane topology of the variant encoded by mRNA-C in comparison with the typical CD9-A tetraspanin structure was predicted on the basis of a hydrophobicity plot (Kyte and Doolittle, 1982) or regional similarity with confirmed transmembrane domains as calculated with the dense alignment surface (DAS) algorithm (Cserzo et al., 1997).

\section{Results}

\section{Initial analyses of CD9 expression by BT cell lines}

CD9 had been analysed by flow cytometry with a set of anti-CD9 antibodies (NCA1-4) (Deissler et al., 1996) which revealed the existence of two cell lines with very low (BT12Ca) or undetectable (BT8Ca) cell surface expression. In accordance with FACS analyses, immunofluorescence staining with the antibody NCA-1 confirmed strong membrane-localized expression of CD9 in BT4Ca cells, weak expression by BT12Ca cells and a lack of CD9-specific immunoreactivity by BT8Ca cells. With mAb MEM-61, preferentially recognizing intracellular CD9, however, CD9 was detected also in BT8Ca cells (Figure 1). In these, the presence of CD9 transcripts was confirmed by RT-PCR, but specific PCR products were not observed when one of the primers was located in exon 1 of the CD9 gene, suggesting transcripts lacking exon 1 or containing an alternative exon 1 (Supplementary Material Figure $\mathrm{S} 1)$.

\section{Identification of two novel CD9 transcripts}

By the RACE technique and subsequent sequencing of the extended 5'-regions, two novel rat $\mathrm{CD} 9$ transcripts with an alternative exon 1 (rCD9 mRNA-B, GenBank accession number $\mathrm{KC} 162232$ ) or beginning with an extended exon 2 (rCD9 mRNA-C, KC162233) were identified in mRNA isolated from BT8Ca cells (Figure 2). The mRNA variant $\mathrm{B}$ lacks a potential translation initiation codon and there is a stop codon in frame with the following CD9 sequence impeding translation into a variant protein. In the 5 '-extension of exon 2, characteristic of mRNA-C, the ORF is not interrupted and an ATG close to the 5'-end suggested efficient translation which was confirmed by expression of a fusion protein with C-terminal GFP in BT4Ca cells. Bright green fluorescence of the transfectant cells indicated translation, which was not observed when mRNA-
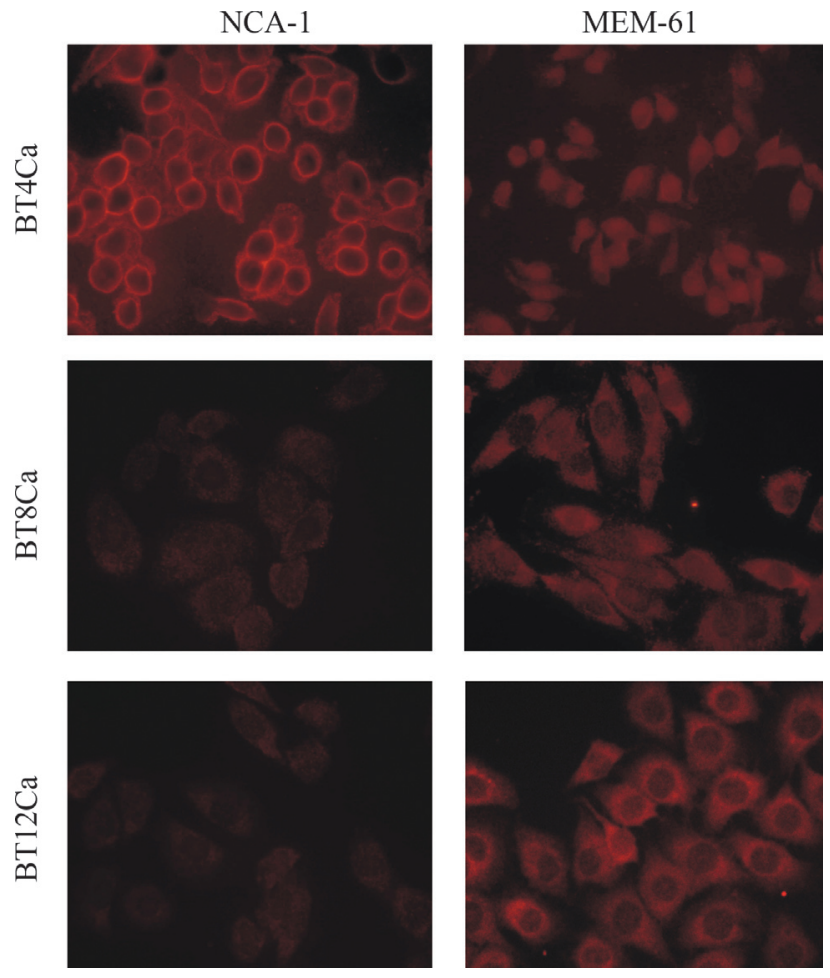

Figure 1 - Immunofluorescence staining with anti-CD9 antibodies. Rat neural cell lines BT4Ca, BT8Ca and BT12Ca were stained with anti-CD9 monoclonal antibodies NCA-1 or MEM-61. Although cell lines BT8Ca and BT12Ca were considered to express only negligible amounts of CD9, specific intracellular staining was observed with antibody MEM-61.

B was coupled to EGFP, or when a construct with a stop codon terminating the $\mathrm{CD} 9$ sequence was used (Figure 3A). The rCD9-C-EGFP fusion protein was also seen as a specific band of expected size when cell lysates were analysed by Western blot with an anti-EGFP antibody (Figure 3B, Supplementary Material Figure S2).

\section{Differential expression of CD9 mRNA variants}

RT-PCR with primers specific for the different CD9 mRNA variants was used to analyse their expression by BT cell lines (Supplementary Material Figure S3). BT4Ca cells strongly expressed all variants, BT8Ca cells did so moderately for mRNA-B and weakly for mRNA-C. BT12Ca and $\mathrm{BT} 13 \mathrm{Ca}$ cells showed the typical strong expression of mRNA-A, but additionally, mRNA-C was expressed. CD9 mRNA-C was also found to be co-expressed with mRNA-A in various tissues (brain, liver, lung, mammary gland, kidney, placenta) of normal Spraque-Dawley rats (Supplementary Material Figure S4), whereas mRNA-B was not detected in any of the tested normal rat tissues.

\section{Discussion}

Our analyses confirmed the atypical CD9 expression by BT8Ca cells in which only traces of the almost ubiquitously expressed transcript mRNA-A were detected. In these cells, two novel transcripts lacking the normal exon 1 

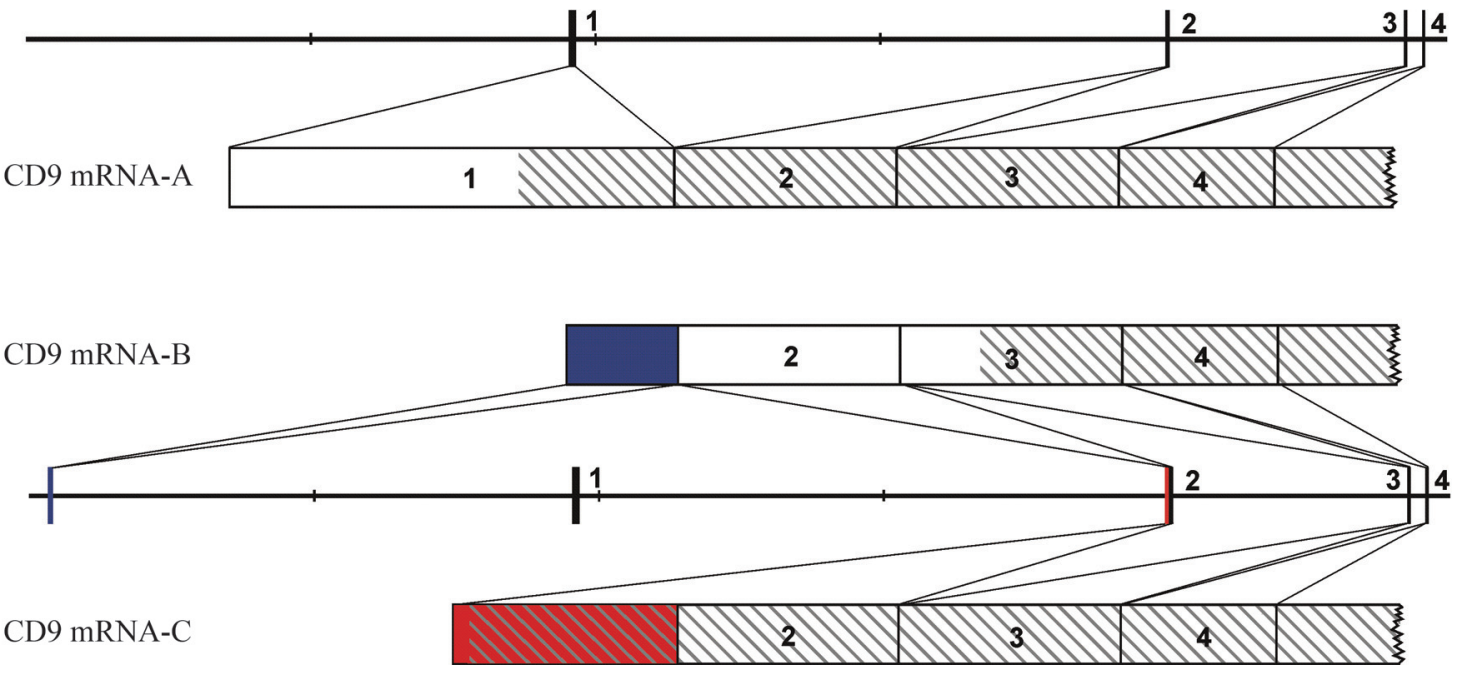

Figure 2 - Positions of exons 1-4 of the different CD9 mRNA variants in the rat genome. Variant mRNA-B is characterized by an alternative exon 1 located 18,618 bp upstream of exon 1 of mRNA-A. mRNA-C contains a prolonged exon 2 with a translation initiation codon. Open reading frames beginning with ATG start codons are indicated by hatched areas. Of the rat chromosome 4, a segment of $50 \mathrm{~kb}$ is shown.
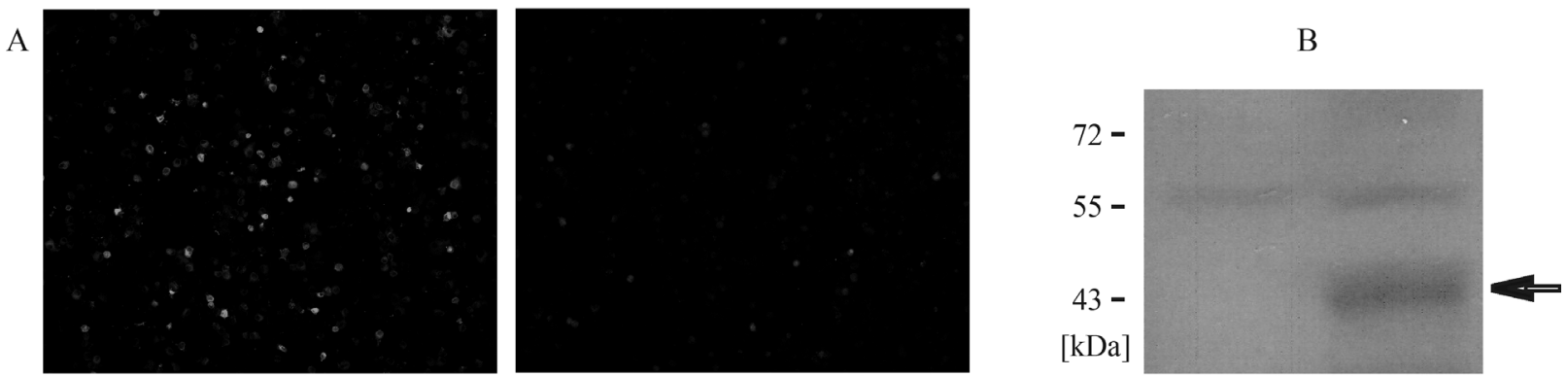

Figure 3 - Expression of a CD9-C-EGFP fusion protein in BT4Ca cells. To confirm that from the ATG located in the extension of exon 2 of CD9 mRNA-C a protein can be translated, the variant CD9 sequence was inserted in a vector to express a fusion protein with C-terminal EGFP only if a functional start codon was provided. For all constructs, three parallel transfections were performed leading to undistinguishable results. (A) Bright fluorescence indicated expression of a fusion protein after transfection of BT4Ca cells (left). In a control transfection, a construct in which the CD9 stop codon had not been removed was used (right). (B) Lysates from transfectant cells were analysed by Western blot with an anti-EGFP antibody recognizing the fusion protein (right lane) which was not detected in cells transfected with the control plasmid (left lane).

were identified. Such aberrant variants have not been recognized previously, most likely due to dominant expression of variant A. mRNA-B seems to be a specific characteristic of the tumorigenic BT cell lines derived by in vitro carcinogenesis from immature rat brain cells (Laerum et al., 1977). Although mRNA-B cannot be translated to a CD9like protein, it may play a role in the regulation of gene expression, e.g. by competing for RNA-binding proteins, as a source of small regulatory RNA fragments (Costa, 2007; Rother and Meister, 2011) affecting specific CD9 expression or even general transcriptional activity through potential effects on DNA methyltransferases (Veeck and Esteller, 2010). In contrast to CD9 mRNA-B, variant $C$ was found accompanying mRNA-A in most of the analysed tissues. Interestingly, the alternative exon 1 of this form contains a functional translation initiation codon and can contribute to the total amount of CD9 proteins. Like many other tetraspanins, CD9 often appears as a double band or even multiple bands when analysed by gel electrophoresis, and it seems possible that, in addition to posttranslational modification, alternative splicing may contribute to this heterogeneity. According to hydrophobicity plots and model calculations to reveal potential transmembrane domains based on the DAS algorithm (Cserzo et al., 1997), the CD9 form translated from mRNA-C should possess an additional transmembrane segment close to the $\mathrm{N}$-terminal end, resulting in a small second intracellular loop and replacement of the $\mathrm{N}$-terminus to the extracellular space (Figure 4). Consequences of such structural differences most likely include an altered connection of CD9-containing protein complexes to the intracellular signalling machinery (Stipp et al., 2003; Levy and Shoham, 2005) and/or a modified spectrum of other proteins binding to CD9. Subsequent studies are needed to reveal specific functions and the relative importance of this CD9 variant. These will be initiated 

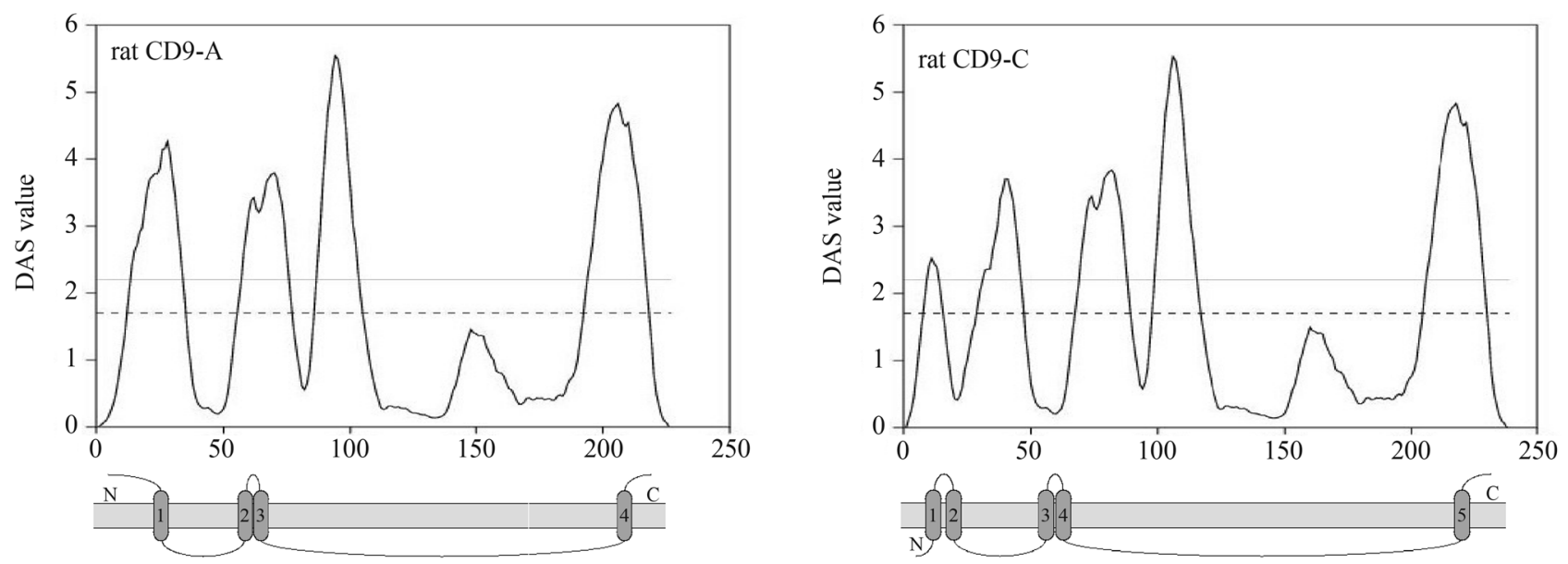

Figure 4 - Predicted membrane topologies of CD9 variants A and C. Hydrophobicity plots and calculations base on the dense alignment surface (DAS) algorithm (upper parts of the subfigures) suggested an additional transmembrane domain encoded by the extended exon 2 of variant CD9-C. Horizontal lines indicate DAS threshold values to define transmembrane domains with low and high stringencies.

by the generation of antibodies binding to the region defined by the alternative exon 1 .

\section{References}

Anton ES, Hadjiargyrou M, Patterson PH and Matthew WD (1995) CD9 plays a role in Schwann cell migration in vitro. $\mathrm{J}$ Neurosci 15:584-595

Costa FF (2007) Non-coding RNAs: Lost in translation? Gene 386:1-10

Cserzo M, Wallin E, Simon I, von Heijne G and Elofsson A (1997) Prediction of transmembrane alpha-helices in prokaryotic membrane proteins: The dense alignment surface method. Protein Eng 10:673-676.

Deissler H, Blass-Kampmann S, Kindler-Rohrborn A, Meyer HE and Rajewsky MF (1996) Characterization of rat NCA/CD9 cell surface antigen and its expression by normal and malignant neural cells. J Neurosci Res 43:664-674.

Hemler ME (2005) Tetraspanin functions and associated microdomains. Nat Rev Mol Cell Biol 6:801-811.

Higashiyama M, Taki T, Ieki Y, Adachi M, Huang CL, Koh T, Kodama K, Doi O and Miyake M (1995) Reduced motility related protein-1 (MRP-1/CD9) gene expression as a factor of poor prognosis in non-small cell lung cancer. Cancer Res 55:6040-6044.

Ishibashi T, Ding L, Ikenaka K, Inoue Y, Miyado K, Mekada E and Baba H (2004) Tetraspanin protein CD9 is a novel paranodal component regulating paranodal junctional formation. J Neurosci 24:96-102.

Kaprielian Z, Cho KO, Hadjiargyrou M and Patterson PH (1995) CD9, a major platelet cell surface glycoprotein, is a ROCA antigen and is expressed in the nervous system. J Neurosci 15:562-573.

Kawashima M, Doh-ura K, Mekada E, Fukui M and Iwaki T (2002) CD9 expression in solid non-neuroepithelial tumors and infiltrative astrocytic tumors. J Histochem Cytochem 50:1195-1203.

Kyte J and Doolittle RF (1982) A simple method for displaying the hydropathic character of a protein. J Mol Biol 157:105132 .
Laerum OD, Rajewsky MF, Schachner M, Stavrou D, Haglid KG and Haugen A (1977) Phenotypic properties of neoplastic cell lines developed from fetal rat brain cells in culture after exposure to ethylnitrosourea in vivo. Z Krebsforsch Klin Onkol Cancer Res Clin Oncol 89:273-295.

Levy S and Shoham T (2005) Protein-protein interactions in the tetraspanin web. Physiology (Bethesda) 20:218-224.

Maecker HT, Todd SC and Levy S (1997) The tetraspanin superfamily: Molecular facilitators. FASEB J 11:428-442.

Miyake M, Nakano K, Itoi SI, Koh T and Taki T (1996) Motility-related protein-1 (MRP-1/CD9) reduction as a factor of poor prognosis in breast cancer. Cancer Res 56:1244-1249.

Mori M, Mimori K, Shiraishi T, Haraguchi M, Ueo H, Barnard GF and Akiyoshi $\mathrm{T}$ (1998) Motility related protein 1 (MRP1/CD9) expression in colon cancer. Clin Cancer Res 4:1507-1510.

Nakamura Y, Iwamoto R and Mekada E (1996) Expression and distribution of CD9 in myelin of the central and peripheral nervous systems. Am J Pathol 149:575-583.

Romanska HM and Berditchevski F (2011) Tetraspanins in human epithelial malignancies. J Pathol 223:4-14.

Rother S and Meister G (2011) Small RNAs derived from longer non-coding RNAs. Biochimie 93:1905-1915.

Sauer G, Windisch J, Kurzeder C, Heilmann V, Kreienberg R and Deissler H (2003) Progression of cervical carcinomas is associated with down-regulation of CD9 but strong local reexpression at sites of transendothelial invasion. Clin Cancer Res 9:6426-6431.

Stipp CS, Kolesnikova TV and Hemler ME (2003) Functional domains in tetraspanin proteins. Trends Biochem Sci 28:106112.

Terada N, Baracskay K, Kinter M, Melrose S, Brophy PJ, Boucheix C, Bjartmar C, Kidd G and Trapp BD (2002) The tetraspanin protein, $\mathrm{CD} 9$, is expressed by progenitor cells committed to oligodendrogenesis and is linked to beta1 integrin, CD81, and Tspan-2. Glia 40:350-359.

Tole S and Patterson PH (1993) Distribution of CD9 in the developing and mature rat nervous system. Dev Dyn 197:94-106.

Veeck J and Esteller M (2010) Breast cancer epigenetics: From DNA methylation to microRNAs. J Mammary Gland Biol Neoplasia 15:5-17. 
Wang HX, Li Q, Sharma C, Knoblich K and Hemler ME (2011) Tetraspanin protein contributions to cancer. Biochem Soc Trans 39:547-552.

\section{Internet Resources}

Hydrophobicity plot software, http://www.expasy.org(cgi-bin/protscale.pl).

Dense alignment surface (DAS) algorithm, http://www.sbc.su.se/ miklos/DAS.

\section{Supplementary Material}

The following online material is available for this article:
- Figure S1 - RT-PCR analyses of CD9 expression by rat BT cell lines

- Figure S2 - Expression of a CD9-B-EGFP fusion protein in BT4Ca cells.

- Figure S3 - Differential expression of CD9 transcript variants by rat $\mathrm{BT}$ cell lines.

- Figure S4 - CD9 mRNA-C is expressed in various rat tissues.

This material is available as part of the online article from http://www.scielo.br/gmb.

Associate Editor: Anamaria Aranha Camargo

License information: This is an open-access article distributed under the terms of the Creative Commons Attribution License, which permits unrestricted use, distribution, and reproduction in any medium, provided the original work is properly cited. 

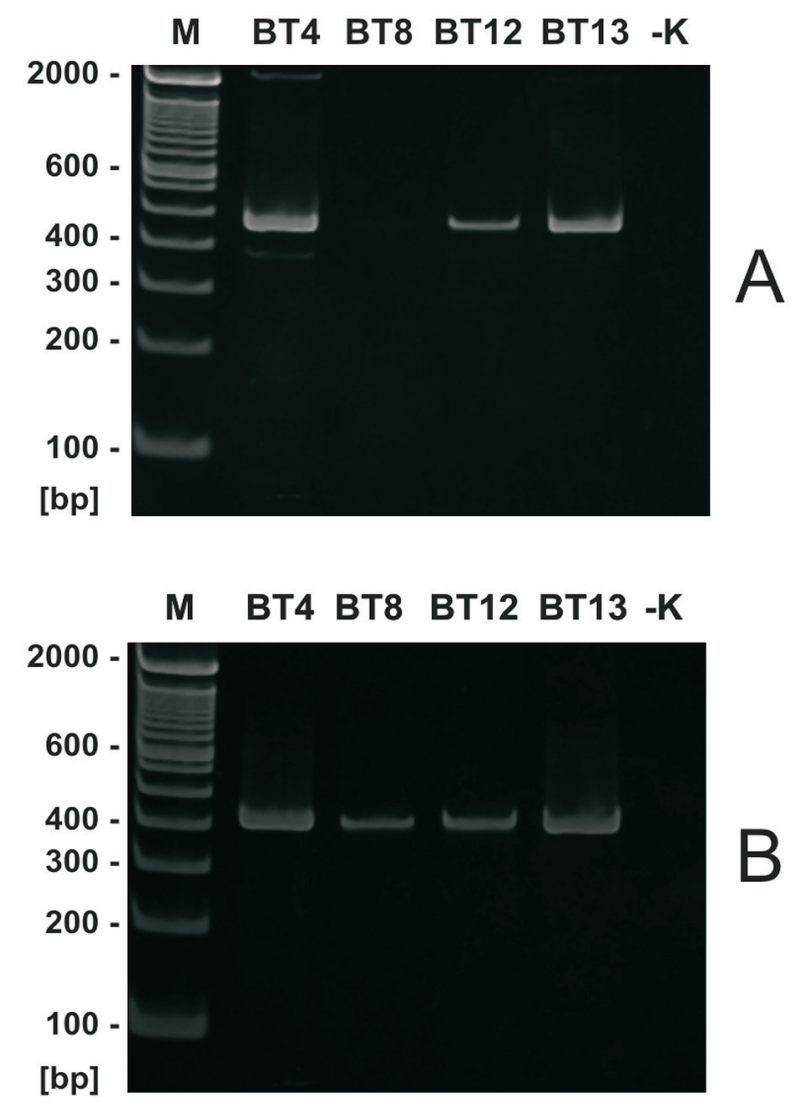

Figure S1 - RT-PCR analyses of CD9 expression by rat BT cell lines. cDNAs were prepared from RNAs expressed by different rat BT cell lines and used as templates in subsequent PCRs with primer pairs covering the first exons (A) or the last exons (B) of the CD9 gene. Lack of a specific PCR product suggested that BT8Ca cells predominantly express a CD9 transcript with a different 5 '-end. 


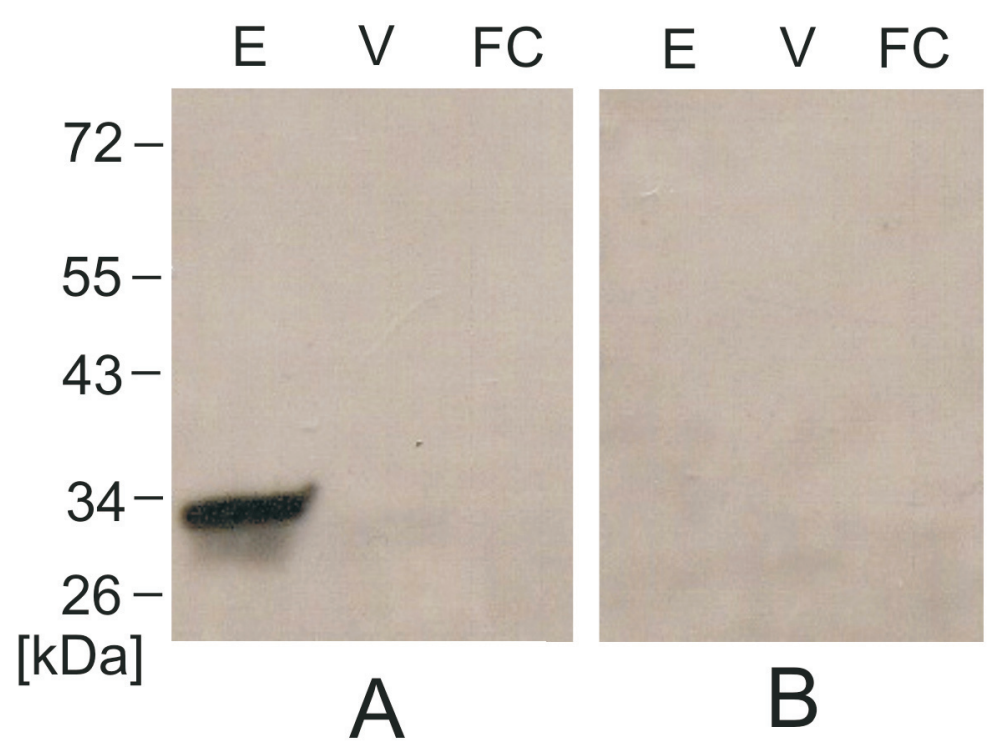

Figure S2 - Expression of a CD9-B-EGFP fusion protein in BT4Ca cells. To test if ratCD9 mRNA-B can be translated, the variant CD9 sequence was inserted in a vector to potentially express a fusion protein with C-terminal EGFP. Lysates from transfectant cells were analysed by Western blot with an anti-EGFP antibody (A). Processing the membrane with an isotype-matched control antibody (B) confirmed specific immunoreactivity.

E: Transfection with a control vector encoding EGFP.

$\mathrm{V}$ : Transfection with vector plasmid without insert.

FC: Transfection with a plasmid construct allowing expression of a mRNA-B-EGFP fusion protein.

In accordance with the lack of an obvious translation initiation codon in the mRNA-B sequence, a 3'-EGFP fusion protein was not detected. 

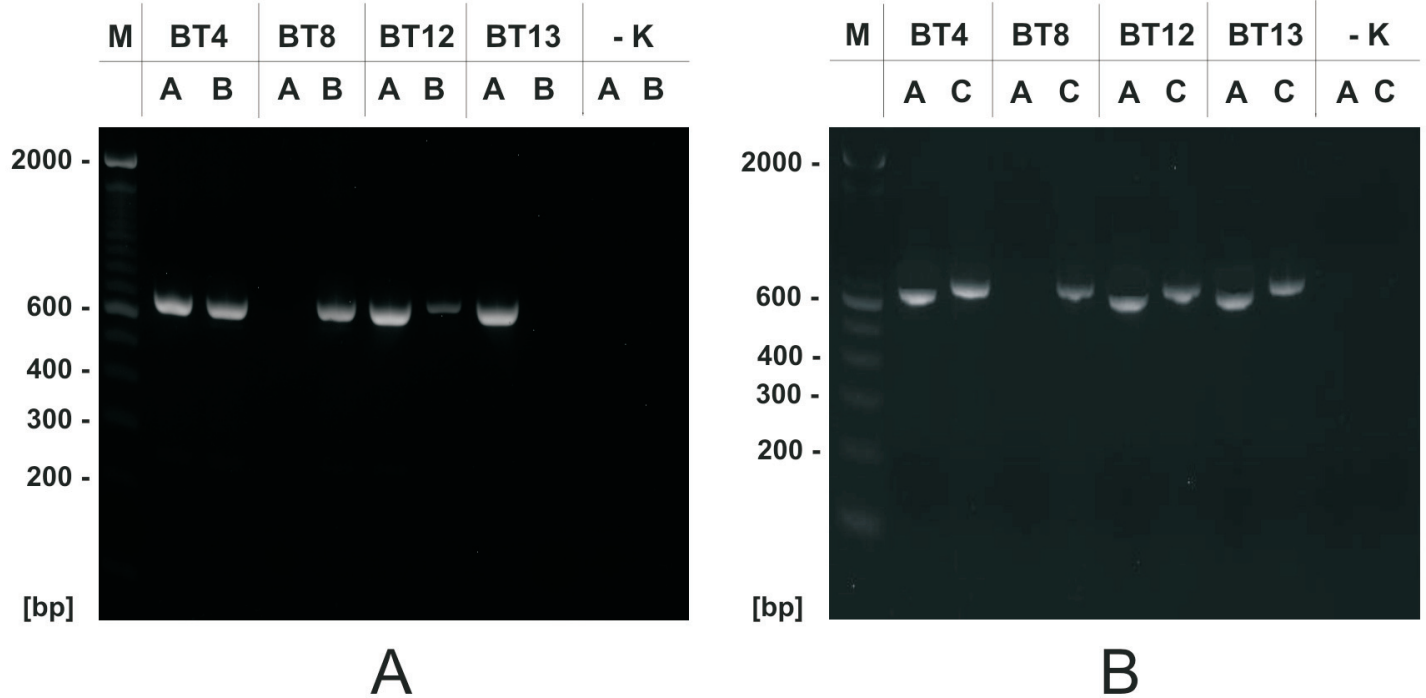

Figure S3 - Differential expression of CD9 transcript variants by rat BT cell lines. cDNAs were prepared from RNAs expressed by different rat BT cell lines and used as templates in subsequent PCRs with primer pairs of which one was specific for CD9 transcripts A,B, or C. The identified novel variants B and $\mathrm{C}$ were separately compared with mRNA-A. It was confirmed that BT8Ca cells lack CD9 mRNA-A but express both transcripts B and C. 


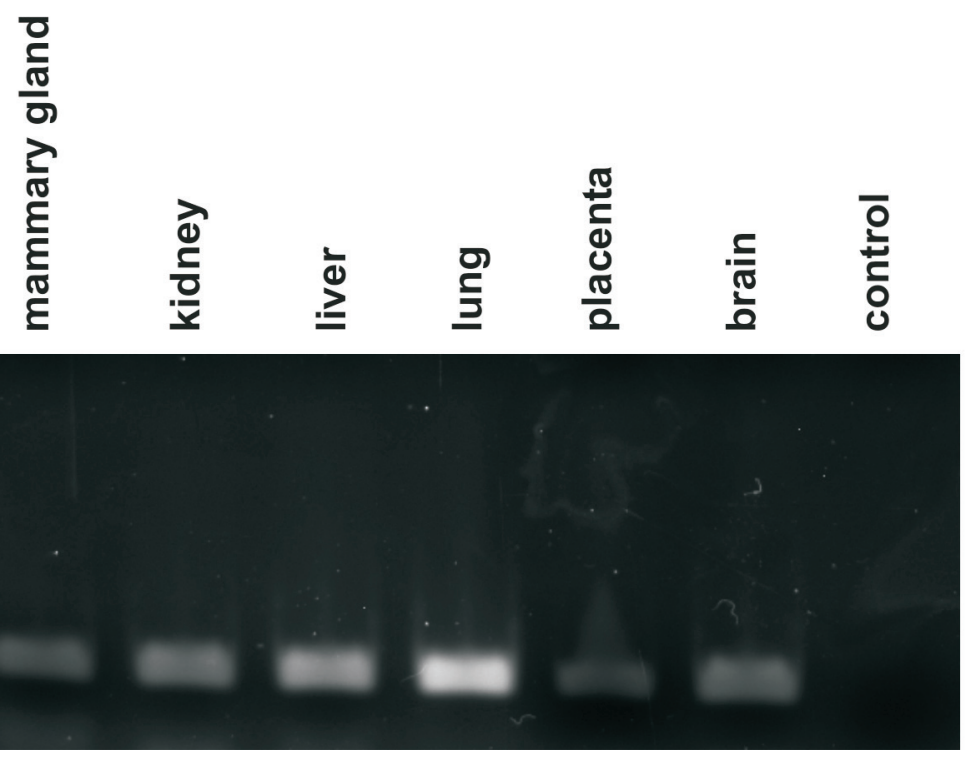

[bp]

Figure S4 - CD9 mRNA-C is expressed in various rat tissues. cDNAs were prepared from RNAs derived from different tissues of normal Spraque-Dawley rats and used as templates in subsequent PCRs with a forward primer specific for CD9 variant C. 